\title{
QUAL O VALOR DA ANÁLISE SEMI-QUANTITATIVA DOS EXAMES DE FDG-PET NO DIAGNÓSTICO DA ESCLEROSE MESIAL TEM PORAL?
}

\author{
Paulo S. Duarte ${ }^{1}$, Hongming Zhuang ${ }^{1}$, Olivier Couturier ${ }^{1}$, Abass Alavi ${ }^{1}$
}

\begin{abstract}
RESUMO - O objetivo deste estudo foi comparar a análise visual dos exames de FDG-PET com uma análise semi-quantitativa no diagnóstico da esclerose mesial temporal (EMT). M étodos: Este estudo incluiu 21 pacientes com confirmação histopatológica de EMT. Os dados referentes à análise visual foram obtidos dos relatórios dos exames. As imagens de FDG-PET foram analisadas de forma semi-quantitativa utilizando-se regiões de interesse (ROIs) desenhadas em 19 cortes tomográficos perpendiculares ao maior eixo do lobo temporal. Estas ROls dividiram cada um dos lobos temporais em três regiões (lateral, inferior e medial). Um índice de assimetria foi calculado para cada região. Resultados: A análise visual dos exames de FDG-PET demonstrou assimetria no metabolismo em todos os pacientes. Todos, menos 1 dos pacientes foram submetidos a lobectomia temporal do tipo standard do lado descrito como hipometabólico pelo exame de FDG-PET. Utilizando como critério um índice de assimetria maior ou igual a $9 \%$ em ao menos uma das regiões, a análise semi-quantitativa demonstrou assimetria considerada significativa em 18 pacientes. Esta assimetria coincidiu com o lado da cirurgia e foi confirmada como correspondente à área de eslerose em todos menos um dos pacientes (o mesmo paciente citado acima). Conclusão: A análise semi-quantitativa não forneceu qualquer informação adicional em relação à interpretação visual das imagens nesta série de pacientes.
\end{abstract}

PALAVRAS-CHAVE: FDG, PET, esclerose mesial temporal, epilepsia.

\section{Does semi-quantitative analysis of FDG-PET have any additional value in the diagnosis of mesial temporal sclerosis?}

\begin{abstract}
The aim of this study was to compare qualitative visual analysis with semi-quantitative analysis in the diagnosis of mesial temporal sclerosis (MTS) using FDG-PET. Methods: This study included 21 patients with histopathological confirmation of MTS. FDG-PET visual analysis data were based on clinical reports generated soon after the completion of the scan. FDG-PET images were semi-quantitatively analyzed using regions of interest (ROIs) in 19 slices perpendicular to the longest axis of the temporal lobe. These ROls divided each temporal lobe into three regions (lateral, inferior and medial). An asymmetry index was calculated for each region. Results: The visual analysis of the FDG-PET studies demonstrated asymmetric hypometabolism in all patients. All but 1 patient underwent standard lobectomy of the same side described as hypometabolic by the PET report. Using an asymmetry index equal or greater than $9 \%$ in at least one of the regions as a threshold, the FDG-PET semi-quantitative analysis showed significant asymmetry in 18 patients. These also matched the side of lobectomy and were confirmed as sclerotic in all but one patient (same patient as above). Conclusion: The semi-quantitative analysis did not provide additional information over visual interpretation in this series of patients.
\end{abstract}

KEY WORDS: FDG, PET, mesial temporal sclerosis, epilepsy.

A cirurgia tem conquistado grande importância no tratamento de pacientes com crise parcial complexa que não apresentam boa resposta ao tratamento medicamentoso ${ }^{1}$. Localizar o foco epiléptico de forma precisa é fundamental para o procedimento cirúrgico. Esta localização pode ser feita utilizando técnicas de eletrencefalografia (EEG) e de neuroimagem como a ressonância nuclear magnética, a tomo- grafia por emissão de fóton único (SPECT) e, quando disponível, a tomografia por emissão de pósitrons $(\mathrm{PET})^{2}$. Os pacientes com epilepsia de origem no lobo temporal constituem o maior grupo a ser submetido a cirurgia. Para que o resultado da cirurgia seja satisfatório éfundamental que o lobo temporal acometido seja corretamente localizado. Para isto, utiliza-se avaliação em conjunto dos métodos diagnós-

'Divisão de Medicina Nuclear, Hospital da Universidade da Pensilvânia, Filadélfia, Pensilvânia, USA. Dr. Hongming Zhuang foi parcialmente apoiado pelo NIH training grant CA-65442-02.

Recebido 1 Maio 2001, recebido na forma final 26 Junho 2001. Aceito 17 Julho 2001.

Dr. Paulo Schiavom Duarte - Rua Cincinato Braga 282 - 01333-910 São Paulo SP - Brasil. FAX: 284 1352. E-mail: paulo.duarte@ fleury.com.br 
ticos citados acima, e a cirurgia só é realizada quando existe boa concordância entre os métodos.

Uma das metodologias que vem ganhando importância na localização do foco epiléptico em pacientes com crise parcial complexa e que se suspeita ter origem em um dos lobos temporais é a tomografia por emissão de pósitrons utilizando como radiofármaco um análogo radioativo da glicose ${ }^{18}$ FDG-Fluordeoxiglicose (FDG-PET) -, durante a fase inter-ictal ${ }^{3-5}$.

A PET é um método diagnóstico que cria imagens tridimensionais e tomográficas da distribuição no organismo de radioisótopos emissores de pósitrons. Os emissores de pósitrons são radioisótopos (ou radionuclídeos) que decaem (passam de um estado de maior energia para um estado de menor energia) pela emissão de pósitron (partícula beta mais $-\beta+$ ), um elétron positivo. O pósitron é classificado como antimatéria, isto é, não existe normalmente na matéria, aparecendo em situações especiais de forma fugaz. De todos os radiofármacos desenvolvidos utilizando emissores de pósitrons, o que mais tem sido utilizado na prática clínica é a FDG, um análogo radioativo da glicose, como já referido acima, e que tem comportamento celular muito parecido a esta última: é internalizada pelas proteínas de membrana GLUT 1 a GLUT 5, é posteriormente fosforilada de FDG para FDG-6P pela hexoquinase a primeira enzima da via glicolítica - e, diferentemente da glicose-6P, não é utilizada pela enzima seguinte, a glicose-6-isomerase, como substrato. Por essa razão, a FDG-6P acumula continuamente na célula enquanto há disponibilidade sanguínea. O único modo para a FDG-6P deixar a célula é sua transformação novamente em FDG pela enzima glicose-6-fosfatase (uma fosforilase). Desta forma, a FDG tem propiciado a realização de "mapas" do consumo de glicose no organismo, assim como o estudo do metabolismo da glicose em alguns órgãos.

Em neurologia, a utilização da FDG tem permitido a avaliação de alterações do metabolismo cerebral presentes em algumas patologias, destacandose quadros epilépticos e quadros demenciais. Em pacientes epilépticos, o hipometabolismo observado em um dos lobos temporais éconsiderado típico para a presença de foco nessa localização e vem complementar as informações obtidas pelos outros exames utilizados na definição do diagnóstico final de esclerose mesial temporal $(E M T)^{3,4}$.

Como regra geral, a análise das imagens dos exames de FDG-PET é realizada de forma qualitativa com uma definição estritamente subjetiva das áreas consideradas hipometabólicas. A análise visual tem se mostrado satisfatória para este propósito, sendo que a maioria dos centros diagnósticos utiliza somente esta técnica na avaliação das imagens. Um dos problemas com os critérios puramente subjetivos é a queda na precisão do método quando as imagens são avaliadas por observador menos experiente.

A utilização de técnicas semi-quantitativas pode vir a representar maneira de diminuir a subjetividade do método sem uma queda na sua acurácia. No entanto, o uso destes recursos na prática clínica é controverso e alguns pesquisadores questionam a sua utilidade clínica ${ }^{6,7}$.

Neste estudo, comparamos os resultados obtidos utilizando uma técnica de análise semi-quantitativa com aqueles obtidos pela análise visual das imagens de FDG-PET, na localização do lobo temporal acometido em uma série de pacientes com EMT.

\section{MÉTODO}

\section{Pacientes}

Este estudo retrospectivo, realizado na Universidade da Pensilvânia -EUA, incluiu 21 pacientes ( 9 homens, 12 mulheres; com idade variando de 18 a 44 anos; idade média de 30,5 anos; 18 destros e 3 canhotos). Todos tinham crise parcial complexa refratária à terapia e foram selecionados para tratamento cirúrgico após avaliação extensa que incluía EEG de superfície e estudos de neuroimagem (PET e ressonância magnética). Todos foram submetidos a lobectomia temporal do tipo standard (lobectomia temporal anterior envolvendo a ressecção do polo temporal, amígdala e hipocampo) baseados nos resultados obtidos nesses estudos, e tiveram confirmação histopatológica de EMT.

\section{Métodos}

Um estudo de FDG-PET inter-ictal foi realizado antes da cirurgia. Nem os pacientes, nem os familiares referiram episódios de convulsão em período inferior a 6 horas da realização do exame. Os pacientes foram injetados com ${ }^{18} \mathrm{FDG}$-Fluordeoxiglicose (1.3M Bq $-4.2 \mathrm{MBq} / \mathrm{Kg}$ ) em um quarto escuro, com os olhos abertos e os ouvidos não oclusos. Eles foram observados por um tecnólogo experiente no período após a injeção até a realização das imagens (aproximadamente 60 minutos), a fim de excluir a possibilidade de crises nesta fase do estudo. Os pacientes foram mantidos durante todo o tempo com o seu esquema de medicação usual. As imagens foram adquiridas usando o HEAD PENN PET ou o PENN PET 240H scanners. Ambos os instrumentos utilizam cristais de iodeto de sódio (Nal(T)) para a detecção de raios $\gamma$ e operam em um modo tridimensional sem septos. O tamanho do voxel para ambos os sistemas é $2 \times 2 \times 2 \mathrm{~mm}$ em todos os 3 planos e a resolução espacial é $5,5 \mathrm{~mm}$ e 3,7 $\mathrm{mm}$ para o PENN PET $240 \mathrm{H}^{8}$ e HEAD PENN PET ${ }^{9}$, respectivamente. As imagens foram processadas usando o programa PETView em uma estação de trabalho Sun. 


\section{Análise dos dados}

Os resultados da avaliação visual dos exames de FDGPET foram obtidos dos laudos finais dos exames. Estes laudos foram feitos por pelo menos um médico nuclear experiente, acompanhado dos residentes do setor de medicina nuclear, logo após a aquisição dos exames. As imagens foram laudadas diretamente da tela do computador utilizando-se diversas escalas de cor e intensidades de cinza. No momento do laudo, os médicos tinham acesso aos resultados dos outros métodos diagnósticos utilizados e ao quadro clínico do paciente.

As análises semi-quantitativas foram realizadas utilizando-se imagens não filtradas mas corrigidas para atenuação. Após reorientação obliqua, foram realizados cortes ( $2 \mathrm{~mm}$ de espessura) perpendicularmente ao maior eixo do lobo temporal. Seis regiões de interesse (ROI) foram desenhadas por um único investigador em cada uma de 19 cortes do lobo temporal, se iniciando a $1 \mathrm{~cm}$ do polo anterior e se estendendo posteriormente. Estas ROIs dividiram os lobos temporais em três partes de cada lado (lateral, medial e inferior) (Fig 1). Isso resultou em 6 grupos de regiões, a saber: temporal lateral esquerda, temporal lateral direita, temporal inferior esquerda, temporal inferior direita, temporal medial esquerda e temporal medial direita.

A contagem total de eventos radioativos e o número de total de pixels em cada região foram somados com aqueles das regiões correspondentes em todos os 19 cortes feitos nos lobos temporais. Em seguida, a soma das contagens dos eventos radioativos em cada grupo de regiões foi dividida pela soma do número de pixels dentro do mesmo grupo de regiões para obter-se, desta forma, a contagem média por pixel em cada grupo de regiões.

As contagens por pixel para os grupos de regiões homólogos nos lobos temporais direito e esquerdo foram comparados usando um índice de assimetria (IA), calculado através da equação: [(R-L)/(R+L)] x 200 (Tabela 1); onde R é a contagem média por pixel no grupo de regiões do lado direito e Lé a contagem média por pixel no grupo de regiões homólogo no lobo temporal esquerdo.

Pela análise semi-quantitativa foi considerada significativa toda assimetria superior ou igual a $9 \%$, em valor absoluto; assimetrias inferiores a 5\%, em valores absolutos, não foram consideradas significativas. Estes valores foram baseados em dados prévios obtidos de literatura. Henry et al. ${ }^{10}$ mostraram que os índices de assimetria nas regiões temporais de voluntários normais são 1,2\% $\pm 4,6 \%$ ( média \pm desvio padrão) para a região temporal lateral e $2,0 \% \pm 4,1 \%$ para a região temporal medial, enquanto pacientes com epilepsia de origem no lobo temporal apresentam índices de assimetria iguais a 13,8\% $\pm 7,0 \%$ e $11,0 \% \pm 8,6 \%$ para a região lateral e medial, respectivamente. Desta forma, exames que apresentavam pelo menos um dos três índices de assimetria maior ou igual a 9\% foram considerados positivos. Exames com todos os três índices inferiores a 5\% foram considerados negativos. Exames que não preencheram nenhum dos dois critérios citados foram classificados como indeterminados.

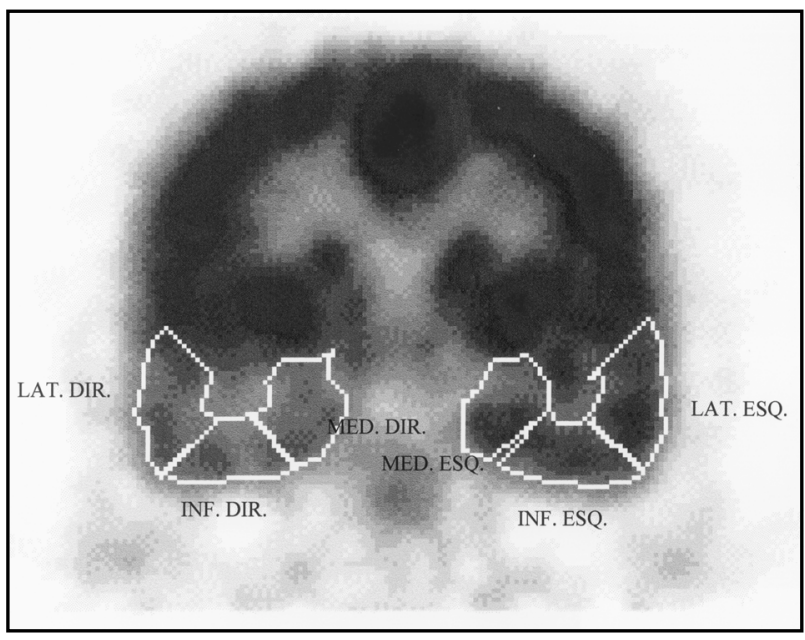

Fig 1. Cortes coronais do cérebro perpendiculares ao longo eixo do lobo temporal demonstrando a divisão de cada lobo temporal nas suas porções lateral, medial e inferior.

Tabela 1. Índices de assimetria nos grupos de regiões lateral, medial e inferior dos lobos temporais para cada um dos 21 pacientes analisados. Em destaque estão os pacientes que apresentaram índices de assimetria inferiores a $5 \%$ em todos os três grupos de regiões.

\begin{tabular}{|c|c|c|c|}
\hline & Lateral & Medial & Inferior \\
\hline Paciente 1 & $-13,8$ & $-4,0$ & $-10,8$ \\
\hline Paciente 2 & 0,8 & $-4,6$ & $-1,7$ \\
\hline Paciente 3 & $-18,9$ & $-3,9$ & $-16,9$ \\
\hline Paciente 4 & $-9,2$ & $-4,7$ & $-5,3$ \\
\hline Paciente 5 & 26,1 & 14,1 & 25,9 \\
\hline Paciente 6 & $-98,2$ & $-20,9$ & $-54,6$ \\
\hline Paciente 7 & 3,3 & 0,5 & 2,5 \\
\hline Paciente 8 & 39,7 & 12,7 & 42,9 \\
\hline Paciente 9 & $-19,7$ & $-18,2$ & $-22,1$ \\
\hline Paciente 10 & $-4,9$ & 3,1 & 1,3 \\
\hline Paciente 11 & $-5,1$ & $-10,5$ & $-9,5$ \\
\hline Paciente 12 & $-4,8$ & 9,6 & 5,0 \\
\hline Paciente 13 & $-14,7$ & $-1,1$ & $-11,3$ \\
\hline Paciente 14 & 8,3 & 3,5 & 10,0 \\
\hline Paciente 15 & 13,2 & $-0,5$ & 16,2 \\
\hline Paciente 16 & $-11,3$ & $-4,5$ & $-5,6$ \\
\hline Paciente 17 & $-14,3$ & $-11,2$ & $-14,6$ \\
\hline Paciente 18 & 8,7 & 3,2 & 16,1 \\
\hline Paciente 19 & $-15,4$ & $-15,5$ & $-8,7$ \\
\hline Paciente 20 & 12,4 & 9,3 & 16,1 \\
\hline Paciente 21 & $-18,6$ & $-14,8$ & $-21,3$ \\
\hline Média* & 13,8 & 7,9 & 13,6 \\
\hline Desvio padrão & 9,2 & 5,7 & 9,9 \\
\hline
\end{tabular}

*As médias foram calculadas a partir dos valores absolutos. 


\section{RESULTADOS}

A análise visual dos exames de FDG-PET demonstrou assimetria no metabolismo em todos os pacientes. Exceto um dos pacientes, todos os demais foram submetidos a lobectomia temporal do tipo standard do lado descrito como hipometabólico pelo exame de FDG-PET. Um paciente foi submetido a lobectomia do lado contralateral aquele demonstrado como hipometabólico pelo FDG-PET, devido ao resultado dos outros métodos diagnósticos utilizados e ao quadro clínico. O resultado do exame anátomo-patológico demostrou esclerose mesial temporal neste caso.

Utilizando-se o critério de positividade descrito previamente, a análise semi-quantitativa demonstrou assimetria considerada significativa em 18 pacientes. O lado considerado hipometabólico coincidiu com o lado da cirurgia e foi confirmado como correspondente a área de eslerose em 17 pacientes (o mesmo paciente citado na análise qualitativa foi submetido a lobectomia do lado contralateral). Assimetria significativa não foi demonstrada em 3 pacientes que tiveram estudos considerados positivos pela análise visual e com posterior confirmação pelo exame histopatológico (estes pacientes apresentaram índices de assimetria inferiores a 5\% em todos os três grupos de regiões). Nenhum dos exames foi classificado como indeterminado pelos critérios utilizados.

\section{DISCUSSÃO}

A possibilidade de realizar-se quantificações nos exames de medicina nuclear faz com que exista uma tendência a tentar quantificar ou semi-quantificar as imagens em busca de maior acurácia e reprodutibilidade dos resultados.

Na área de tomografia por emissão de pósitrons (PET) esta tendência é ainda mais perceptível e atualmente muitas técnicas de semi-quantificação, como o Standard Uptake Value (SUV), têm sido desenvolvidas. Isto se deve, em parte, às origens da PET, que foi utilizada inicialmente como instrumento de investigação científica nas mais diversas áreas da Medicina, com destaque especial para a neurologia. DiChiro e colaboradores ${ }^{11}$, em seus trabalhos pioneiros, associaram a captação do FDG nos tumores cerebrais ao seu grau histológico, utilizando para isto técnicas quantitativas.

Esses trabalhos pioneiros criaram a impressão de que o uso de técnicas quantitativas pudessem trazer maior acurácia para os exames de FDG-PET, e muitos têm tentado utilizá-las na prática clínica.
Atentos a este fato, aqueles mesmos pesquisadores que utilizaram intensamente as técnicas quantitativas nos seus projetos de investigação científica, começaram a criticar a utilização destas metodologias na prática clínica. O próprio DiChiro, em editorial para o "Journal of Nuclear Medicine", há alguns anos $^{7}$, discorre sobre isto, e conclui que na prática clínica o médico deve basear-se menos nos números que na sua interpretação subjetiva e no bom senso na realização do laudo.

Apesar de todas as ressalvas levantadas aos métodos quantitativos, alguns ainda acreditam no seu valor na prática clínica etentam utilizá-los na definição do laudo.

Este estudo, que avaliou o valor de um método semi-quantitativo na localização do foco epiléptico em pacientes com crise parcial complexa e confirmação pós-operatória de esclerose mesial temporal, parece corroborar a opinião daqueles que não acreditam no valor destes métodos na análise dos exames de FDG-PET na prática clínica.

Em conclusão, nesta série de pacientes, a técnica de análise semi-quantitativa utilizada não foi superior à interpretação visual das imagens na definição do lado acometido pela esclerose mesial temporal.

Agradecimentos - Os autores gostariam de agradecer a Flávia Aldighieri pela sua análise crítica desse trabalho.

\section{REFERÊNCIAS}

1. Falconer MA, Taylor DC. Surgical treatment of drug-resistant epilepsy dueto mesial temporal sclerosis: etiology and significance. Arch N eurol 1968;19:353-361.

2. Spencer SS. Therelativecontributions of MRI, SPECT, and PET imaging in epilepsy. Epilepsia 1994;35(Suppl 6):S72-S89.

3. Engel J Jr., Brown WJ, Kuhl DE, Phelps ME, Mazziotta JC, Crandall $\mathrm{PH}$. Pathological findings underlying focal temporal lobehypometabolism in partial epilepsy. Ann Neurol 1982;12:518-528.

4. Mazziotta JC, Engel J Jr. The use and impact of positron computed tomography scanning in epilepsy. Epilepsia 1984;25(Suppl 2):S86-104.

5. Sackellares JC, Siegel GJ, A bou-Khalil BW, et al. Hutchins GD. Differences between lateral and mesial temporal metabolism interictally in epilepsy of mesial temporal origin. Neurology 1990;40:1420-1426.

6. Keyes JW Jr. SUV: standard uptake or silly useless value? J Nucl Med 1995;36:1836-1839.

7. DiChiro G, Brooks RA. PET quantitation: blessing and curse [editorial] [published erratum appears in J Nucl Med 1989;30:129]. J Nucl Med 1988;29:1603-1604.

8. Karp JS, Muehllehner G. Standards for performance measurements of PET scanners: evaluation with theUGM PENN-PET 240H scanner. Med Progr Technol 1991;17:173-187.

9. Karp JS, Freifelder R, Geagan MJ, et al. Three-dimensional imaging characteristics of the HEAD PENN-PET scanner. J Nucl Med 1997; 38:636-643.

10. Henry TR, Mazziotta JC, Engel J Jr., et al. Quantifying interictal metabolic activity in human temporal lobe epilepsy. J Cer Blood Flow Metab 1990;10:748-757.

11. Di Chiro G, DeLaPaz RL, Brooks RA, et al. Glucose utilization of cerebral gliomas measured by [18F] fluorodeoxyglucose and positron emission tomography. Neurology 1982;32:1323-1329. 